\title{
The expression of small RNAs in exosomes of follicular fluid altered in human polycystic ovarian syndrome
}

\author{
Junhe Hu ${ }^{\text {Corresp., } 1}{ }^{\text {, Tao Tang }}{ }^{1}$, Zhi Zeng ${ }^{1}$, Juan Wu ${ }^{1}$, Xiansheng Tan ${ }^{1}$, Jiao Yan ${ }^{1}$ \\ ${ }^{1}$ Agriculture and Biotechnology Department, Hunan University of Humanities, Science and Technology, Loudi, Hunan province, China \\ Corresponding Author: Junhe $\mathrm{Hu}$ \\ Email address: 260477247@qq.com
}

Polycystic ovary syndrome (PCOS) can cause reproductive disorders that may affect oocyte quality from punctured follicles in human follicular fluid (HFF). The non-coding RNA family includes micro RNA (miRNA), piwi-interacting RNA (piRNA) and transfer RNA (tRNA); these non-coding RNA transcripts play diverse functions and are implicated in a variety of diseases and health conditions, including infertility. In this study, to explore the role of HFF exosomes in PCOS, we extracted and sequenced RNA from HFF exosomes of PCOS patients and compared the analysis results with those of non-PCOS control group. The HFF exosomes were successfully isolated and characterized in a variety of ways. The sequencing results of the HFF exosomal RNA showed that about $6.6 \%$ of valid reads in the PCOS group and $8.6 \%$ in the non-PCOS group were successfully mapped to the human RNA database. Using hierarchical clustering method, we found there were ten small RNA sequences whose expression was significantly different between the PCOS and non-PCOS groups. We chose 6 of them to predict target genes of interest for further GO analysis, and pathway analysis showed that the target genes are mainly involved in biosynthesis of amino acids, glycine, serine and glycosaminoglycan, as well as threonine metabolism. Therefore, the small RNA sequences contained in HFF EXs may play a key role in the mechanism that drives PCOS pathogenesis, thereby can act as molecular biomarkers for PCOS diagnosis in the future. 
1

2

3

4

5

6

The expression of small RNAs in exosomes of follicular fluid altered in human polycystic ovarian syndrome

3
Junhe Hu*, Tao Tang, Zhi Zeng, Juan Wu, Tan Xiansheng, Yan Jiao (Agriculture and Biotechnology Department, Hunan University of Humanities, Science and Technology, Loudi, Hunan 417000 )

*Corresponding Author:

Junhe $\mathrm{Hu}$

$8 \quad$ Road Dingxing 7\#, Loudi City, HuNan Province, 417000, China

Email address: 260477247@qq.com or junhe_hu@126.com

0

This manuscript has been thoroughly edited by a native English speaker from an editing company. Editing Certificate will be provided upon request.

Abstracts: Polycystic ovary syndrome (PCOS) can cause reproductive disorders that may affect oocyte quality from punctured follicles in human follicular fluid (HFF). The non-coding RNA family includes micro RNA (miRNA), piwi-interacting RNA (piRNA) and transfer RNA (tRNA); these non-coding RNA transcripts play diverse functions and are implicated in a variety of diseases and health conditions, including infertility. In this study, to explore the role of HFF exosomes in PCOS, we extracted and sequenced RNA from HFF exosomes of PCOS patients and compared the analysis results with those of non-PCOS control group. The HFF exosomes were successfully isolated and characterized in a variety of ways. The sequencing results of the HFF exosomal RNA showed that about $6.6 \%$ of valid reads in the PCOS group and $8.6 \%$ in the non-PCOS group were successfully mapped to the human RNA database. Using hierarchical clustering method, we found there were ten small RNA sequences whose expression was significantly different between the PCOS and non-PCOS groups. We chose 6 of them to predict target genes of interest for further GO analysis, and pathway analysis showed that the target 
27 genes are mainly involved in biosynthesis of amino acids, glycine, serine and 28 glycosaminoglycan, as well as threonine metabolism. Therefore, the small RNA sequences 29 contained in HFF EXs may play a key role in the mechanism that drives PCOS pathogenesis, 30 thereby can act as molecular biomarkers for PCOS diagnosis in the future.

32 Keywords: Exosomes (EXs); human follicular fluid (HFF); polycystic ovary syndrome (PCOS); 33 piRNAs; miRNAs 


\section{Introduction}

At present, studies have found that a diverse array of hormones (FSH, LH, statins, activins and estrogens), growth factors, peptides, proteins and nucleic acids are present in human follicular fluid (HFF), and these substances help establish a unique microenvironment for the growth and maturation of oocytes [1]. HFF is initially derived from the blood in blood vessels in the wall of the follicle. During development, the follicle has extensive contact with various types of adjacent cells to maintain the proper growth and development of the oocyte [2]. Studies have demonstrated that follicular somatic cells can have contact with each other, mainly through the transport of extracellular vesicles (EVs), which play important regulatory roles that can affect oocyte maturation. Therefore, it is likely that a large number of EVs are present in the follicular fluid in order to transport these communication signals effectively. HFF is a complex mixture of proteins, metabolites, and ionic compounds and its composition dictates the general state of follicular metabolism and therefore the maturation and quality of oocytes.

EVs are cell-derived vesicles found in the majority of eukaryotic fluids, including HFF, blood, and cell culture media. EVs can consist of several different vesicle structures and are either continuously secreted by cells or produced under certain stimulatory conditions. EVs can be broadly categorized as exosomes (EXs), microvesicles (MVs), and apoptotic vesicles (AVs) [3]. Generally, EVs are classified according to their size, with EXs ranging from 30-150 nm in size, MVs ranging from 150-1000 $\mathrm{nm}$ in size, and AVs ranging from 1-5 mm in size [15]. Moreover, studies have found that EVs can contain a large number of biologically active molecular substances such as proteins, mRNAs and miRNAs, which can be released from cells via the plasma membrane by budding and fission to exert their effects on neighboring cells [4]. EXs are formed by the invagination of multivesicular bodies in the cell which then fuse to the plasma membrane and are then released outside the cell [1]. EXs are typically released from vesicular structures in the endoplasmic reticulum under normal physiological conditions and are released through the plasma membrane to exert regulatory functions on nearby cells[5]. The trafficking of EXs and their contents plays an important role in regulation of cell physiological 
62

63

64

65

66

functions $[2,6]$.

It has been previously reported that EXs exist in horse [2] and human [7]follicular fluid under normal physiological conditions and in some cases under abnormal physiological conditions, such as in patients with polycystic ovary syndrome [8-10] and milk[11]. Recently, studies have reported that miRNAs can regulate the expression of various genes during oocyte maturation and follicular growth [12]. Several studies have reported that miRNAs are not only present in cells but also in plasma, serum, urine, saliva and milk, where they play diverse and essential biological regulatory roles $[8,13,14]$. Additional studies have found that extracellular miRNAs are abundantly and stably present in plasma and possess high ribonuclease activity [15] and that these miRNAs are contained within EXs and therefore protected from their external environment [16]. miRNAs are small regulatory RNA molecules (typically around 22 nucleotides long) that modulate posttranscriptional gene regulation by binding to specific mRNA targets $[16,17]$. These miRNAs play essential roles in a wide range of physiological processes, many of which have a critical role in female fertility and the female reproductive system (follicular development and oocyte maturation) [18]. There have been several reports that miRNAs are present in HFF[2, 19, 20], but there are no existing reports regarding HFF EXs that contain non-coding small RNA transcripts.

Polycystic ovary syndrome (PCOS) is condition that is the result of elevated androgens (male hormones) in females (approximately $5-10 \%$ of all women of reproductive age are affected). Signs and symptoms of PCOS can include irregular or no menstrual periods, heavy periods, excess body and facial hair, acne, pelvic pain, difficulty getting pregnant, and patches of thick, darker, velvety skin[21]. A previous study found that the circulating level of miRNA-21 was significantly increased in PCOS patients, which resulted in decreased expression of MST1/2, LATS1/2, TAZ in PCOS patients when compared to control subjects[19]. This study also showed that miR-132 and miR-320 were expressed at significantly lower levels in HFF from PCOS patients when compared to healthy controls[22]. Roth et al. found that 29 miRNAs were significantly differentially expressed between PCOS and healthy control patient samples. Out of 
89

90

91

92

93

94

95

the 29 miRNAs identified in this study, hsa-miR-9, hsa-miR-18b, hsa-miR-32, hsa-miR-34c and hsa-miR-135a expression were significantly increased in PCOS follicular fluid samples compared to controls[10]. Only miR-132 and miR-320 were found to be expressed at a significantly lower level in PCOS patients when comparing the miRNAs found in the follicular fluid of PCOS patients against that of healthy controls[22]; this contrasts the findings regarding higher expression of miR-320 in PCOS patients in another study[23]. The expression levels of three miRNAs, miR-222, miR-146a and miR-30c have been reported to be significantly increased in PCOS patients compared to controls; this finding was validated by the finding that miR-222 expression is positively associated with serum insulin levels while miR-146a expression is negatively associated with serum testosterone levels[24]. Although some studies have reported alterations in MiRNA expression in PCOS, there are no existing reports regarding HFF EXs that contain non-coding small RNA transcripts and PCOS or reports of MiRNAs that may be promising candidates for biomarkers for PCOS diagnosis and treatment.

It is likely that EXs and their contents, such as miRNAs, play a vital regulatory role in HFF during oocyte growth and development. Therefore, it is necessary to compare the expression levels of non-coding small RNA transcripts in HFF-derived EXs from PCOS and healthy patients. The findings of this study revise and improve our understanding of the content of HFFderived EXs, thus laying the foundation for the future investigation of the role of miRNAs in PCOS pathogenesis.

\section{Materials and methods}

\section{Follicular fluid sample collection}

The patients that donated the follicular fluid samples used in this study were undergoing conventional IVF treatment. Moreover, all experiments were approved by the Institutional Ethics Committee of Hunan University of Humanities, Science and Technology (\#20180308). Informed consent was obtained from each couple regarding the use of the follicular fluid sample that was obtained during IVF treatment oocyte retrieval for this study, which was approved by 
115 the Institutional Ethics Committee of Shaoyang HuiEn Reproductive and Health Hospital 116 (\#20180316). The form is attached in a supplemental file. The current diagnostic criterion for

117 PCOS is based on the revised 2003 criteria (two out of three is enough for positive diagnosis) as 118 following: (a) oligo-ovulation and/or anovulation; (b) clinical and/or biochemical signs of 119 hyperandrogenism; and (c) polycystic ovaries.

120 Controls and PCOS patients (patient basic information in Table 1) were injected with 121 recombinant FSH following treatment with $\mathrm{GnRH}$ agonists according to the standard protocol for 122 IVF treatment. FSH stimulation was initiated once downregulation was confirmed by ultrasound 123 and measurements of serum estradiol, luteinizing hormone, and progesterone. Real-time 124 ultrasound scans were used to assess follicular growth at two day intervals from day 5 of FSH treatment until the day of oocyte retrieval. When at least one ovarian follicle had grown to 18-20 $\mathrm{mm}$ in diameter, the ovums were punctured under the guidance of vaginal B-ultrasound after 34-

$12738 \mathrm{~h}$ following hCG trigger.

128

129

130

131

132

133

134

135

136

137

138

139

HFF was collected via transvaginal ultrasound-guided puncture and aspiration of follicles that were 18-20 mm in diameter. HFF was collected from each patient donor. HFF samples were centrifuged at $1300 \mathrm{~g}$ for $15 \mathrm{~min}$ to remove the cells; blood and other material obtained was then stored at $-80^{\circ} \mathrm{C}$ for further experiments.

\section{Exosomes Purification and Characterization}

HFF EXs were purified and characterized according to previously published protocols with some modifications [25]. The EX isolation method was performed as follows: a $15 \mathrm{~mL}$ pooled sample generated from combined HFF from patients was centrifuged at 3,500 rpm for 15 min at $4^{\circ} \mathrm{C}$ to pellet debris. The supernatant was then transferred to a $15 \mathrm{~mL}$ ultracentrifuge tube and ultracentrifuged at $16,500 \mathrm{~g}$ for $30 \mathrm{~min}$ at $4{ }^{\circ} \mathrm{C}$, and then filtered through a $0.2 \mathrm{~mm}$ syringe filter to obtain the medium contained exosomes. Finally, exosomes were pelleted by ultracentrifugation at $120,000 \mathrm{~g}$ for $70 \mathrm{~min}$ at $4^{\circ} \mathrm{C}$ and stored at $-80^{\circ} \mathrm{C}$ for further analysis. 
For nanoparticle tracking analysis (NTA), obtained exosomes were diluted in phosphate-

141

142

143 buffered saline (PBS). Specifically, samples were diluted with sterile PBS according to the manufacturer's instructions. Capture and analysis settings were manually set according to the above clinical protocol. Using a Flow Nano-Analyzer instrument (NanoFCM Inc., Xiamen, China), EXs were visualized with laser light scattering, and Brownian motion of the EXs was captured on video. Recorded videos were further analyzed with Image J computer software, which provided high-resolution particle size distribution profiles and concentration measurements of the EXs in solution.

Flow cytometry analysis was carried out using a previously published protocol [26]. Briefly, stored EX pellets are resuspended with $100 \mathrm{ul}$ of filtered PBS and kept on ice. A negative control sample was prepared without EX staining and labeled NC, and the positive sample was stained with antibodies for CD63 and CD81 and labeled accordingly. Samples and controls were suspended in PBS and analyzed at a FACS workstation (NanoFCM Inc., Xiamen, China) with the guidance of the instrument operating procedures.

EX pellets were suspended in PBS for electron microscopy analysis. First, 5-10 $\mu \mathrm{L}$ of a resuspended EXs pellet is added to a copper mesh and precipitated for $3 \mathrm{~min}$ as filter paper absorbs the volatile liquid from the edge. Second, negative staining with phosphotungstic acid was carried out following a PBS wash. Finally, the mesh was dried at room temperature for 2 min before imaging on the electron microscope operating voltage 80-120 kv (JEM-1200EX, Japan Electronics Co., Ltd).

\section{EX small RNA library construction}

EX pellets were resuspended in Trizol (Invitrogen) for RNA isolation. The small RNA library from EXs was constructed using the total RNA from $15 \mathrm{~mL}$ of pooled HFF. Small RNA cloning, sequencing, and analysis were carried out as described previously using the QIAseq ${ }^{\circledR}$ miRNA Library Kit [22]. RNA detection and quality control was carried out using an Agilent 2100 Bio-analyzer (Agilent Technologies Sweden AB). 
166

167

168

169

170

171

172

173

174

175

176

177

178

179

180

181

182

183

184

185

186

187

188

189

190

\section{RNA-seq data analysis of EXs from human follicular fluids}

Initially, the sequencing adaptors and low-complexity reads were removed in an initial data filtering step, and the quality of reads was estimated with the FASTQC program. Reads were aligned against the human reference genome (hg19 February 2009, GRCh37) downloaded from the GENCODE project (http://www.gencodegenes.org/) and used for all subsequent bioinformatic analyses. Finally, we applied a DEBseq-counts algorithm to filter the differentially expressed genes following FDR analysis using the criteria $\log 2 \mathrm{FC}>1$ and $\mathrm{FDR}<0.05$ to choose the significantly expressed genes for further research. Functional annotation was performed using the Database for Annotation, Visualization and Integrated Discovery (DAVID) v6.8 (https://david.ncifcrf.gov). Pathway analysis was carried out using annotated data downloaded from KEGG (https://www.genome.jp/kegg/).

\section{Statistical analysis}

Both the back-spliced junction reads and linear mapped reads were combined and scaled to reads per kilobase per million mapped reads (RPKM) to quantify the expression level of miRNAs, piRNAs, and tRNAs. Differences in expression profiles between the PCOS group and the non-PCOS group were analyzed using a Student's t-test. A $P<0.05$ was considered to indicate statistically significant differences.

\section{Results}

\section{Isolation and Characterization of HFF-derived EXs}

HFF EXs were successfully separated and characterized as described in the methods section. EXs isolated from HFF had an average diameter of $75 \mathrm{~nm}$, which is consistent with the reported range of EX sizes, as shown in Figure 1A. Moreover, CD63 and CD81 are enriched in EXs, and our samples were positive for both, as shown in Figures 1B-F. EX pellets were resuspended in PBS for electron microscopy analysis, as shown in Figures 1G-I, to further confirm the presence of intact EXs. 
191

192

193

194

195

196

197

198

199

200

201

202

203

204

205

206

207

208

209

210

211

212

213

214

215

216

\section{Quality control analysis of RNA-seq data}

FastQC software (https://www.bioinformatics.babraham.ac.uk/projects/fastqc/) was used to perform evaluate the quality of sequenced data, including mass value distribution, position value distribution, and the GC content, as shown in Figure 2. The quality control analysis of the raw sequencing data gives a quick overview of the data before use in further analysis.

\section{Analysis of small RNAs in HFF EXs}

RNA sequencing and analysis were carried out using four EX samples (two prepared from PCOS patients compared against two prepared from non-PCOS patients). On average, the total number of valid reads obtained from the EXs of patients with PCOS (7.50 million) was similar that of the control EXs (6.86 million), as shown in Table 2. Overall, about $6.6 \%$ and $8.6 \%$ of all valid reads from this set of two PCOS and two non-PCOS EX samples, respectively, could be successfully mapped to the human non-coding RNA database and studied further (Table 2).

MiRNAs differentially expressed between the PCOS and non-PCOS samples were further analyzed using hierarchical clustering methods to generate a heatmap comparing the results for the PCOS group (sample P1 and P2) and control group (sample C1 and C2), as shown in Figure 3A. From this analysis comparing the PCOS and control groups, we chose ten significantly upregulated (miR-6087, miR-4745-3p, miR-193b-3p, miR-199a-5p, miR-4532, miR-199a-3p, miR-199b-3p, miR-629-5p, miR-143-3p, and miR-25-3p) and 10 significantly downregulated (miR-98-5p, miR-483-5p, miR-382-5p, miR-23b-3p, miR-10a-5p, miR-200a-3p, miR-141-3p, miR-3911, miR-200c-3p, and miR-483-3p) miRNAs for further study, as shown in Figure 3B. Analysis of differently expressed piRNAs was also performed using hierarchical clustering methods to generate a heatmap comparing the PCOS group (sample P1 and P2) and the control group (sample C1 and C2), as shown in Figure 4A. From this analysis comparing the PCOS and control groups, we chose ten piRNAs with significantly upregulated expression (pir-36441, pir57942, pir-54998, pir-34896, pir-33221, pir-51671, pir-33226, pir-43997, pir-33405, and pir36040) and ten piRNAs with significantly downregulated expression (pir-43772, pir-35414, pir- 
217 43771, pir-35413, pir-35469, pir-33065, pir-35463, pir-35468, pir-35467, and pir-33387) for

218 further study, as shown in Figure 4B. Analysis of differentially expressed tRNAs was also

219 performed using hierarchical clustering methods to generate a heatmap comparing the PCOS

220 group (sample P1 and P2) and the control group (sample C1 and C2), as shown in Figure 5A. we

221 chose ten tRNAs with significantly upregulated expression (tsrna-12365, tsrna-12363, tsrna222 12362, tsrna-12364, tsrna-12361, tsrna-12360, tsrna-12359, tsrna-17099, tsrna-17100, and tsrna-

223 12395) and ten tRNAs with significantly downregulated expression (tsrna-06177, tsrna-06176,

224 tsrna-14935,tsrna-14937, tsrna-15209, tsrna-14934, tsrna-15199, tsrna-15198, tsrna-03939, and

225 tsrna-03940) for further study, as shown in Figure 5B.

226

227

228

229

230

231

232

233

234

235

236

237

238

239

240

241

242

\section{GO and Pathway analysis of miRNA target genes}

Mature miRNAs are produced by a series of nueclease-mediated cleavages of longer primary transcripts, which are then assembled into RNA-induced silencing complexes in order to identify target genes via complementary base pairing. This guides the silencing complexes to degrade target genes or block the translation of target genes according to the degree of complementarity with the miRNA sequence.

Based on the above research results, six miRNAs (miR-6087, miR-199a-5p, miR-143-3p, miR-483-5p, miR-200a-3p, and hsa-miR-23b-3p) were predicted to be relevant to human PCOS when Miranda and RNA software were used to predict the targeting genes regulation relationship (the intersecting results of the two prediction softwares were used as the final list of target gene predictions). The parameters of this analysis were set as follows: energy_miranda $<-20$, score_miranda $>150$, and energy_RNAhybird $<-25$. The results of this analysis reveal 146 significantly different target genes, which are used to take GO further as shown in Figure 6 and pathway analysis as shown in Figure 7.

\section{Discussion}

The follicular follicle in the ovary provides a unique microenvironment for the maturation of oocytes and interactions between follicular somatic cells and oocytes. It is therefore crucial to 
243 dissect the molecular components of HFF in order to elucidate the mechanisms and regulatory 244 processes involved in oocyte maturation. EXs are known to be essential carriers for signal 245 transduction-mediated interaction between follicular somatic cell sand oocytes in order to 246 promote oocyte maturation in $\operatorname{HFF}[27,28]$. In this study, we found that $\operatorname{HFF}$ EXs can be 247 successfully isolated and have an average diameter of $75 \mathrm{~nm}$. The presence of intact isolated EXs 248 was confirmed using transmission electron microscopy and FACS (EXs are positive for CD63 249 and CD81)[29-31].

Additional studies of the miRNAs contained and expressed in HFF EXs are necessary to

251

252

253

254

255

256

257

258

259

260

261

262

263

264

265

266

267

268

269

reveal the mechanisms that promote oocyte maturation and how this process is regulated by the axis of follicular somatic cells-EXs-oocytes. Similarly to Sang et al.[32], we identified potential EX-contained miRNAs and their gene targets using bioinformatics approaches. We found that about $6.6 \%$ of PCOS and $8.6 \%$ of non-PCOS patient EX-contained miRNAs could be successfully mapped to the human miRNA database (Table 2), revealing ten miRNAs with significantly upregulated expression (miR-6087, miR-4745-3p, miR-193b-3p, miR-199a-5p, miR-4532, miR-199a-3p, miR-199b-3p, miR-629-5p, miR-143-3p, and miR-25-3p) and ten miRNAs with significantly downregulated expression (miR-98-5p, miR-483-5p, miR-382-5p, miR-23b-3p, miR-10a-5p, miR-200a-3p, miR-141-3p, miR-3911, miR-200c-3p, and miR-4833p) when comparing results from PCOS and healthy patients. Several of these miRNAs are potentially novel biomarkers for PCOS that have not been mentioned in previous reports[33, 34]. Furthermore, we found a variety of piRNAs and tRNAs are significantly differentially expressed when comparing results from PCOS and healthy patients (Figure 4 and 5). PiRNAs are small RNA molecules that are 24-32 nucleotides long and are abundant in the germline across animal species [35]. A previous study identified twenty-six piRNAs that were differentially expressed in the cumulus cells of diminished ovarian reserve patients compared to controls [36]. We identified ten piRNAs whose expression is either significantly up- or downregulated in PCOS patients compared to controls (Figure 4). Some of these piRNAs could be potential biomarkers for molecular diagnosis of PCOS in the future. Our results also show that ten tRNAs whose 
270

271

272

273

274

275

276

277

278

279

280

281

282

283

284

285

286

287

288

289

290

291

292

293

294

295

296

expression is either significantly different up- or downregulated in PCOS patients compared to controls (Figure 5). It is interesting to note that mitochondrial tRNA mutations have been previously associated with PCOS[37]. Some of these significantly differentially expressed small RNAs could play mechanistic roles PCOS pathogenesis and should be studied farther.

Through our analysis, we identified six miRNAs (miR-6087, miR-199a-5p, miR-143-3p, miR-483-5p, miR-23b-3p and miR-200a-3p) that could be relevant to PCOS pathogenesis. These six MiRNAs underwent target genes analysis, which revealed that miR-483-5p is reported as PCOS biomarker[38], as well as miR-200a-3p[39]. Currently, there is no gold standard for miRNA gene target analysis[40], so we elected to use Miranda and RNA software to take GO analysis and pathways according to reported papers[41]. The results of our analysis show that the relevant miRNA-regulated pathways are mainly related to amino acid biosynthesis, glycine, serine and threonine metabolism, glycosaminoglycan biosynthesis, monocarboxylic acid metabolism, and carbon metabolism. However, these potential miRNA targeted genes and pathways are predicted, not experimentally validated, and therefore the associations observed in this study are limited. Some studies have shown that each miRNA can have hundreds of gene targets and the gene targets may be different based on cell type and the presence or absence of other miRNAs [40]. The impact of a given miRNA on gene expression may be physiologically essential but difficult to identify with bioinformatics alone[42]. To date, there is no diagnostic test for PCOS, only several classes of diagnostic criteria from 3 different entities: the Rotterdam criteria [43], NIH criteria[42], and Androgen Excess and PCOS Society[44]. Consequently, diagnosing PCOS is highly dependent on the symptomatic criteria used[45]. Although PCOS is the most common endocrine disorder in women, few susceptibility genes have been consistently linked with the syndrome[46].

Therefore, our study revealed that various miRNAs, piRNAs and tRNAs are differentially expressed in EXs in the HFF of women with PCOS when compared to controls, and these small RNAs target gene clusters and pathways that correspond to a variety cell functions. Some of these target genes may be potential biomarkers for PCOS diagnosis and treatment. Moreover, 
297 many questions remain unanswered regarding the relationship between MiRNAs in EXs and the 298 mechanisms that drive PCOS, including the following: what is the relationship between the small 299 RNAs (miRNA, piRNA and tRNA) contained in HFF EXs and PCOS pathogenesis? Which 300 miRNAs might promote the development of PCOS? Do small RNAs contained in HFF EXs play 301 a mechanistic role in driving PCOS?

\section{Conclusions}

303 We found that a variety of non-coding small RNAs contained in HFF-derived EXs from 304 PCOS patients are significantly differentially expressed when compared to control patient 305 samples. Some of these differentially expressed small RNAs or their target genes may be 306 potential biomarkers for diagnosis and treatment of PCOS. However, the mechanism that relates 307 HFF EX-contained miRNAs to PCOS pathogenesis remains to be elucidated in future studies. 
309

$310 \quad 1$.

311

312

313

314

315

316

317

318

319

320

321

322

323

324

325

326

327

328

329

330

331

332

333

334

335

336

337

338

339

340

341

342

343

344

345

346

347

348

349

\section{References}

1. Desrochers LM, Bordeleau F, Reinhart-King CA, Cerione RA, Antonyak MA: Microvesicles provide a mechanism for intercellular communication by embryonic stem cells during embryo implantation. Nature communications 2016, 7:11958.

2. da Silveira JC, Veeramachaneni DNR, Winger QA, Carnevale EM, Bouma GJ: Cell-secreted vesicles in equine ovarian follicular fluid contain miRNAs and proteins: a possible new form of cell communication within the ovarian follicle. Biology of reproduction 2012, 86(3):71, 71-10.

3. Raposo G, Stoorvogel W: Extracellular vesicles: exosomes, microvesicles, and friends. J Cell Biol 2013, 200(4):373-383.

4. Muralidharan-Chari V, Clancy JW, Sedgwick A, D'Souza-Schorey C: Microvesicles: mediators of extracellular communication during cancer progression. J Cell Sci 2010, 123(10):1603-1611.

5. Farooqi AA, Desai NN, Qureshi MZ, Librelotto DRN, Gasparri ML, Bishayee A, Nabavi SM, Curti V, Daglia M: Exosome biogenesis, bioactivities and functions as new delivery systems of natural compounds. Biotechnology advances 2017.

6. Di Pietro C: Exosome-mediated communication in the ovarian follicle. Journal of assisted reproduction and genetics 2016, 33(3):303-311.

7. Yamamoto CM, Tovar V, Murakami T, Ogura M, Oakes M, Mitsuhashi M: Methods for collecting cervicalvaginal fluids and isolating exosome and microvesicles for molecular analysis. In.: Google Patents; 2017.

8. Ding C-F, Chen W-Q, Zhu Y-T, Bo Y-L, Hu H-M, Zheng R-H: Circulating microRNAs in patients with polycystic ovary syndrome. Human Fertility 2015, 18(1):22-29.

9. Palomba S, Daolio J, La Sala GB: Oocyte competence in women with polycystic ovary syndrome. Trends in Endocrinology \& Metabolism 2017, 28(3):186-198.

10. Roth LW, McCallie B, Alvero R, Schoolcraft WB, Minjarez D, Katz-Jaffe MG: Altered microRNA and gene expression in the follicular fluid of women with polycystic ovary syndrome. Journal of assisted reproduction and genetics 2014, 31(3):355-362.

11. Sohel MMH, Hoelker M, Noferesti SS, Salilew-Wondim D, Tholen E, Looft C, Rings F, Uddin MJ, Spencer TE, Schellander K: Exosomal and non-exosomal transport of extra-cellular microRNAs in follicular fluid: implications for bovine oocyte developmental competence. PloS one 2014, 8(11):e78505.

12. Hossain MM, Sohel MMH, Schellander K, Tesfaye D: Characterization and importance of microRNAs in mammalian gonadal functions. Cell and tissue research 2012, 349(3):679-690.

13. Murri M, Insenser M, Fern谩ndez-Dur漫n E, San-Mill谩n JL, Luque-Ram铆rez M, Escobar-Morreale HF: Non-targeted profiling of circulating microRNAs in women with polycystic ovary syndrome (PCOS): effects of obesity and sex hormones. Metabolism 2018.

14. Mitchell PS, Parkin RK, Kroh EM, Fritz BR, Wyman SK, Pogosova-Agadjanyan EL, Peterson A, Noteboom J, O'Briant KC, Allen A: Circulating microRNAs as stable blood-based markers for cancer detection. Proceedings of the National Academy of Sciences 2008, 105(30):10513-10518.

15. Van den Brande S, Gijbels M, Wynant N, Santos D, Mingels L, Gansemans Y, Van Nieuwerburgh F, Broeck JV: The presence of extracellular microRNAs in the media of cultured Drosophila cells. Scientific reports 2018, 8(1):17312-17321.

16. Pegtel DM, Cosmopoulos K, Thorley-Lawson DA, van Eijndhoven MAJ, Hopmans ES, Lindenberg JL, de Gruijl TD, W眉rdinger T, Middeldorp JM: Functional delivery of viral miRNAs via exosomes. Proceedings

Peer] reviewing PDF | (2019:09:41331:2:0:NEW 18 Jan 2020) 
of the National Academy of Sciences 2010, 107(14):6328-6333.

17. Andrade GM, Meirelles FV, Perecin F, da Silveira JC: Cellular and extracellular vesicular origins of miRNAs within the bovine ovarian follicle. Reproduction in Domestic Animals 2017, 52(6):1036-1045.

18. Tesfaye D, Gebremedhn S, Salilew-Wondim D, Hailay T, Hoelker M, Grosse-Brinkhaus C, Schellander K: MicroRNAs: tiny molecules with a significant role in mammalian follicular and oocyte development. Reproduction 2018, 155(3):R121-R135.

19. Jiang L, Li W, Wu M, Cao S: Ciculating miRNA-21 as a biomarker predicts polycystic ovary syndrome (PCOS) in patients. Clin Lab 2015, 61:1009-1015.

20. Liu K, Zhang D: The profile of free and exosomal mirnas in follicular fluid of OHSS women. Fertility and Sterility 2018, 110(4):e332.

21. Dewailly D, Hieronimus $S$, Mirakian $\mathrm{P}$, Hugues JN: Polycystic ovary syndrome (PCOS). In: Annales D'endocrinologie: 2010: Elsevier; 2010: 8-13.

22. Sang $Q$, Yao $Z$, Wang $H$, Feng $R$, Wang $H$, Zhao $X$, Xing $Q$, Jin L, He L, Wu L: Identification of microRNAs in human follicular fluid: characterization of microRNAs that govern steroidogenesis in vitro and are associated with polycystic ovary syndrome in vivo. The Journal of Clinical Endocrinology \& Metabolism 2013, 98(7):3068-3079.

23. Yin $M$, Wang $X$, Yao $G$, Lu $M$, Liang $M$, Sun $Y$, Sun $F$ : Transactivation of miR-320 by miR-383 regulates granulosa cell functions by targeting E2F1 and SF-1. Journal of Biological Chemistry 2014:jbc. M113. 546044.

24. Long W, Zhao C, Ji C, Ding H, Cui Y, Guo X, Shen R, Liu J: Characterization of serum microRNAs profile of PCOS and identification of novel non-invasive biomarkers. Cellular Physiology and Biochemistry 2014, 33(5):1304-1315.

25. Cecilia L, Maria E, Jan L: Isolation and Characterization of RNA-Containing Exosomes. Journal of Visualized Experiments Jove 2012, 59(59):e3037.

26. Wahlgren J, Karlson TDL, Brisslert M, Sani FV, Telemo E, Sunnerhagen P, Valadi H: Plasma exosomes can deliver exogenous short interfering RNA to monocytes and lymphocytes. Nucleic Acids Research 2012, 40(17):e130.

27. Cakmak H, Franciosi F, Zamah AM, Cedars MI, Conti M: Dynamic secretion during meiotic reentry integrates the function of the oocyte and cumulus cells. Proceedings of the National Academy of Sciences 2016, 113(9):2424-2429.

28. Van Blerkom J: Molecular Mining of Follicular Fluid for Reliable Biomarkers of Human Oocyte and Embryo Developmental Competence. In: In Vitro Fertilization. Springer; 2019: 929-937.

29. Chen C, Zong S, Wang Z, Lu J, Zhu D, Zhang Y, Cui Y: Imaging and intracellular tracking of cancer-derived exosomes using single-molecule localization-based super-resolution microscope. ACS applied materials \& interfaces 2016, 8(39):25825-25833.

30. Lo Sicco C, Reverberi D, Pascucci L, Tasso R: A Method for Isolating and Characterizing Mesenchymal Stromal Cell-derived Extracellular Vesicles. Current protocols in stem cell biology 2018, 46(1):e55.

31. Yang J, Li Y, Xue F, Liu W, Zhang S: Exosomes derived from cardiac telocytes exert positive effects on endothelial cells. American journal of translational research 2017, 9(12):5375.

32. BRÄNNSTRÖM M, Johansson BM, Sogn J, Janson PO: Characterization of an in vitro perfused rat ovary model: ovulation rate, oocyte maturation, steroidogenesis and influence of PMSG priming. Acta 
Physiologica 2010, 130(1):107-114.

33. Murri M, Insenser M, Fernandez-Duran E, San-Millan JL, Escobar-Morreale HF: Effects of polycystic ovary syndrome (PCOS), sex hormones, and obesity on circulating miRNA-21, miRNA-27b, miRNA-103, and miRNA-155 expression. The Journal of Clinical Endocrinology \& Metabolism 2013, 98(11):E1835-E1844.

34. Sathyapalan T, David R, Gooderham NJ, Atkin SL: Increased expression of circulating miRNA-93 in women with polycystic ovary syndrome may represent a novel, non-invasive biomarker for diagnosis. Scientific reports 2015, 5:16890-16897.

35. Thomson T, Lin H: The biogenesis and function of PIWI proteins and piRNAs: progress and prospect. Annual Review of Cell and Developmental 2009, 25:355-376.

36. Chen D, Zhang Z, Chen B, Ji D, Hao Y, Zhou P, Wei Z, Cao Y: Altered microRNA and Piwi-interacting RNA profiles in cumulus cells from patients with diminished ovarian reserve. Biology of reproduction 2017, 97(1):91-103.

37. Ding $\mathrm{Y}$, Xia B-H, Zhang C-J, Zhuo G-C: Mitochondrial tRNALeu (UUR) C3275T, tRNAGIn T4363C and tRNALys A8343G mutations may be associated with PCOS and metabolic syndrome. Gene 2018, 642:299-306.

38. Shi L, Liu S, Zhao W, Shi J: miR-483-5p and miR-486-5p are down-regulated in cumulus cells of metaphase II oocytes from women with polycystic ovary syndrome. Reproductive biomedicine online 2015, 31(4):565-572.

39. Dhaded S, Dabshetty S: Role of circulation miRNA in patients suffering with polycystic ovary syndrome (PCOS). International Journal of Clinical Obstetrics and Gynaecology 2018, 2(3):92-95.

40. Zhang YF, Zhang R, Su B: Diversity and evolution of MicroRNA gene clusters. Science in China 2009, 52(3):261.

41. Riffo-Campos A, Riquelme I, Brebi-Mieville P: Tools for sequence-based miRNA target prediction: what to choose? International journal of molecular sciences 2016, 17(12):1987.

42. Ricardo A, Enrico C, Didier D, Evanthia DK, Escobar-Morreale HF, Walter F, Janssen OE, Legro RS, Norman RJ, Taylor AE: The Androgen Excess and PCOS Society criteria for the polycystic ovary syndrome: the complete task force report. Fertility \& Sterility 2009, 91(2):456-488.

43. Lauritsen MP, Bentzen JG, Pinborg A, Loft A, Forman JL, Thuesen LL, Cohen A, Hougaard DM, Nyboe AA: The prevalence of polycystic ovary syndrome in a normal population according to the Rotterdam criteria versus revised criteria including anti-Mullerian hormone. Human Reproduction 2014, 29(4):791.

44. Abbott DH, Fida B: Ontogeny of polycystic ovary syndrome and insulin resistance in utero and early childhood. Fertility \& Sterility 2013, 100(1):2-11.

45. Yildiz BO, Bozdag G, Yapici Z, Esinler I, Yarali H: Prevalence, phenotype and cardiometabolic risk of polycystic ovary syndrome under different diagnostic criteria. Human Reproduction 2012, 27(10):3067.

46. Anvesha S, Helle G, Alexander D, Malathi R, Juliet C, Catalin M, Oermann EK, Sunghae U, Kim JS, Chen LN: MicroRNA profiling in prostate cancer--the diagnostic potential of urinary miR-205 and miR-214. Plos One 2013, 8(10):e76994. 
Table $\mathbf{1}$ (on next page)

sample characteristics

Clinical characteristics of PCOS and control subjects 
1 Table 1 Clinical characteristics of PCOS and control subjects

2

\begin{tabular}{ccccc}
\hline Sample Name & P1 & P2 & C1 & C2 \\
\hline BMI $\left(\mathrm{kg} / \mathrm{m}^{2}\right)$ & 24.9 & 26.8 & 23.9 & 26.3 \\
Age (years) & 29 & 40 & 45 & 43 \\
Anti-Müllerian hormone $(\mathrm{ng} / \mathrm{mL})$ & 15.88 & 8.42 & 1.59 & 2.69 \\
Antral Follicle count (number) & 20 & 16 & 17 & 14 \\
Luteinizing hormone (mIU/mL) & 11.6 & 4.25 & 2.57 & 5.1 \\
Follicle stimulating hormone $(\mathrm{mIU} / \mathrm{mL})$ & 5.14 & 5.59 & 4.86 & 5.58 \\
Estradiol (pg/mL) & 51.3 & 42.65 & 31.81 & 33.31 \\
Prolactin (ng/mL) & 11.87 & 6.14 & 14.66 & 8.14 \\
Free testosterone $(\mathrm{ng} / \mathrm{mL})$ & 62.15 & 42.37 & 41.68 & 33.36 \\
\hline
\end{tabular}

3 


\section{Table 2 (on next page)}

sequencing data mapping to the human genome

The results of showing non-coding RNAs sequencing data mapping to the human genome $s$ from HFF exosomes 
1 Table 2 The results of showing non-coding RNAs sequencing data mapping to the human 2 genome s from HFF exosomes

\begin{tabular}{ccccc}
\hline Sample Name & P1 & P2 & C1 & C2 \\
\hline All Reads & 9163276 & 5827574 & 8657970 & 5070271 \\
UnMapped & 8835532 & 5269328 & 7946685 & 4611534 \\
Mapped & 327744 & 558246 & 711285 & 458737 \\
MappedRate & 0.036 & 0.096 & 0.082 & 0.09 \\
UniqueMapped & 250217 & 398550 & 562175 & 344625 \\
UniqueMappedRate & 0.027 & 0.068 & 0.065 & 0.068 \\
RepeatMapped & 77527 & 159696 & 149110 & 114112 \\
\hline
\end{tabular}

3 


\section{Figure 1}

\section{Characterization of of HFF exosomes}

$<$ !--[if !supportLists]-->(A)<!--[endif]-->Nanoparticle tracking measurement of exosomes particle size and concentration was shown. Pelleted fractions from FF are vesicles whose diameter size ranged from 15 to $165 \mathrm{~nm}$, with a peak size between 50 and $100 \mathrm{~nm}$. Average vesicle size was $75 \mathrm{~nm}$. Particle size is consistent with exosome size range. (B) Flow cytometry without checking samples . (C) Flow cytometry picture for exosomes sample without using monoclonal antibodies against the tetras panins CD63. (D) Flow cytometry picture for exosomes sample using monoclonal antibodies against the tetras panins CD63. (E) Flow cytometry picture for exosomes sample without using monoclonal antibodies against the tetras panins CD81. (F) Flow cytometry picture for exosomes sample using monoclonal antibodies against the tetras panins CD81. $\square \mathrm{G} \square$ Transmission electron microscopy of exosomes under the view of 2 um size microscope rulers. (H) Transmission electron microscopy of exosomes under the view of $500 \mathrm{~nm}$ size microscope rulers. (I) Transmission electron microscopy of exosomes under the view of $200 \mathrm{~nm}$ size microscope rulers. 

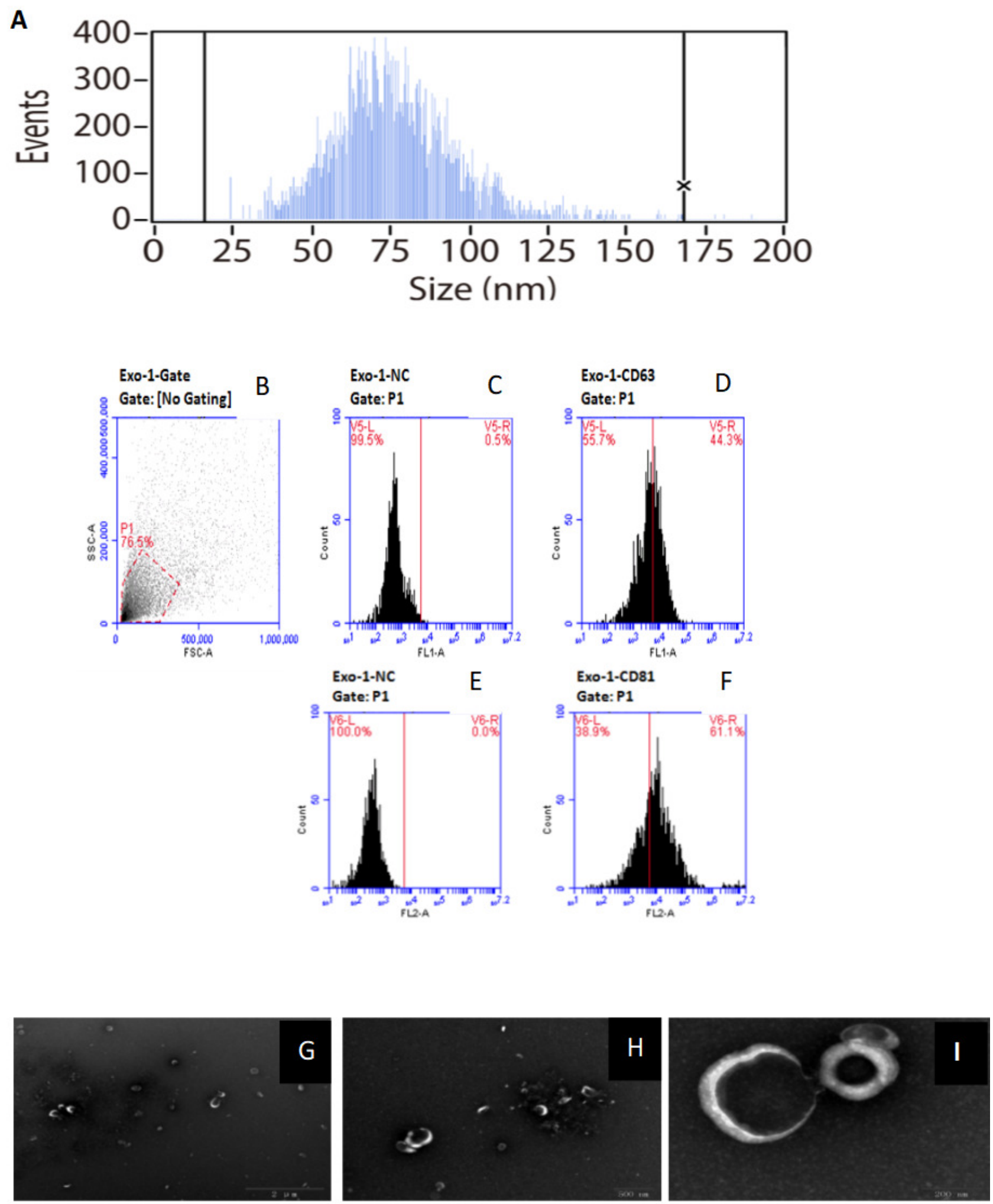


\section{Figure 2}

Quality control of RNA-Seq data of HFF exosomes

(A) The horizontal axis represents the number of bases or the range of the number of bases, and the vertical axis represents the value of the quality score, where Quality scores for 20 indicates that the mapping accuracy is greater than $99 \%$ and can be used for subsequent analysis. (B) The horizontal axis represents average Quality scores and the vertical axis represents reads. The blue line indicates the distribution of theoretical GC content, and the red line indicates the distribution of actual GC content.

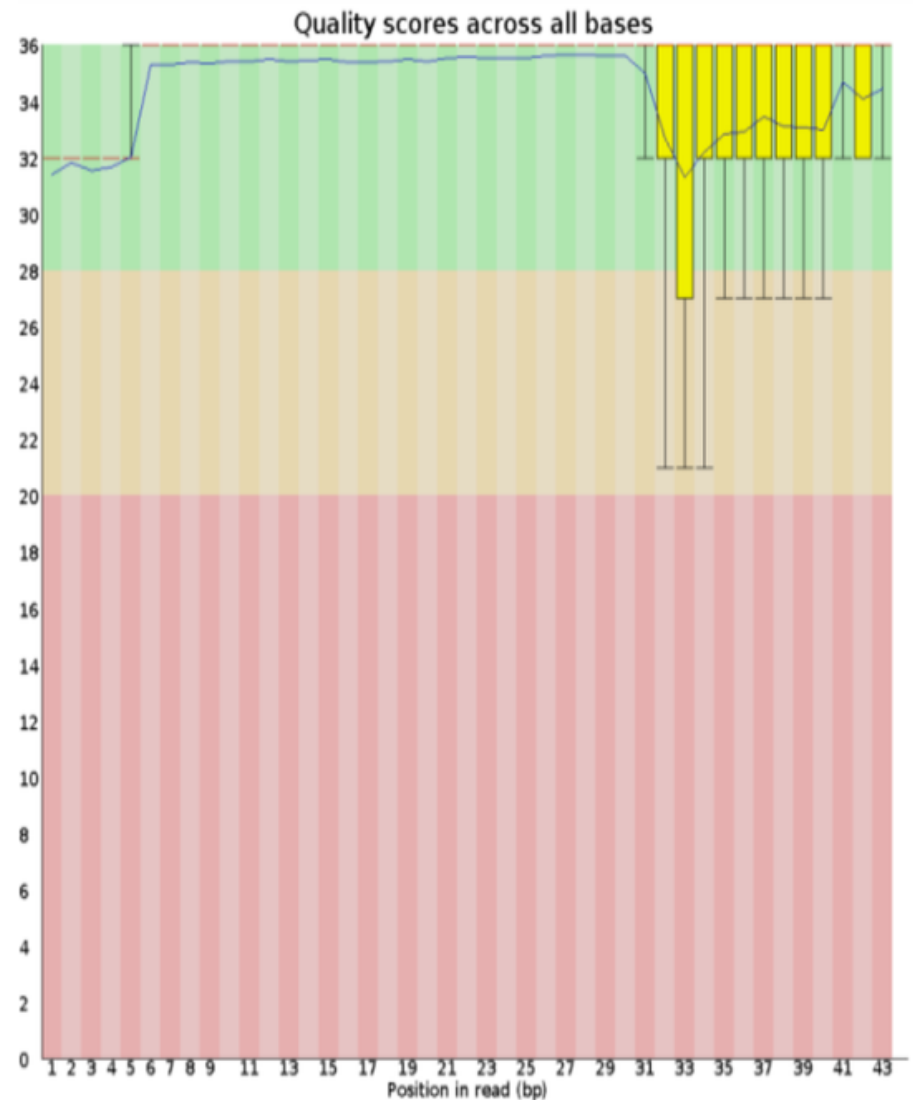

A

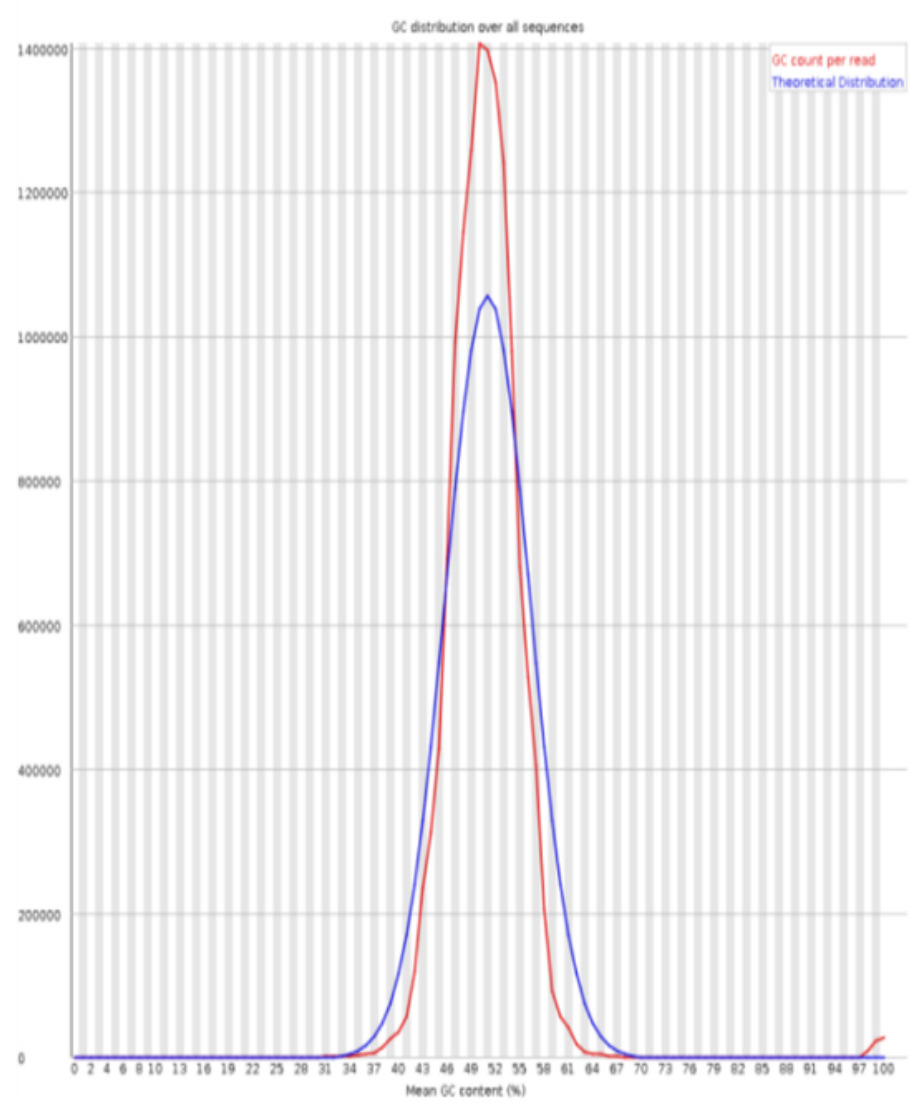

B 


\section{Figure 3}

The miRNA expression analysis of HFF exosome

$S(A)$ Heat map of hierarchical clustering analysis of miRNA shown in HFF of PCOS group (P1 and P2) and the control group (C1 and C2). (B) These are 10 significantly up and down expression miRNA separately according to their $P$ Value and fold changes $(P<0.05$ and log $2 \mid($ fold-changes) $\mid>1$ ) 
A
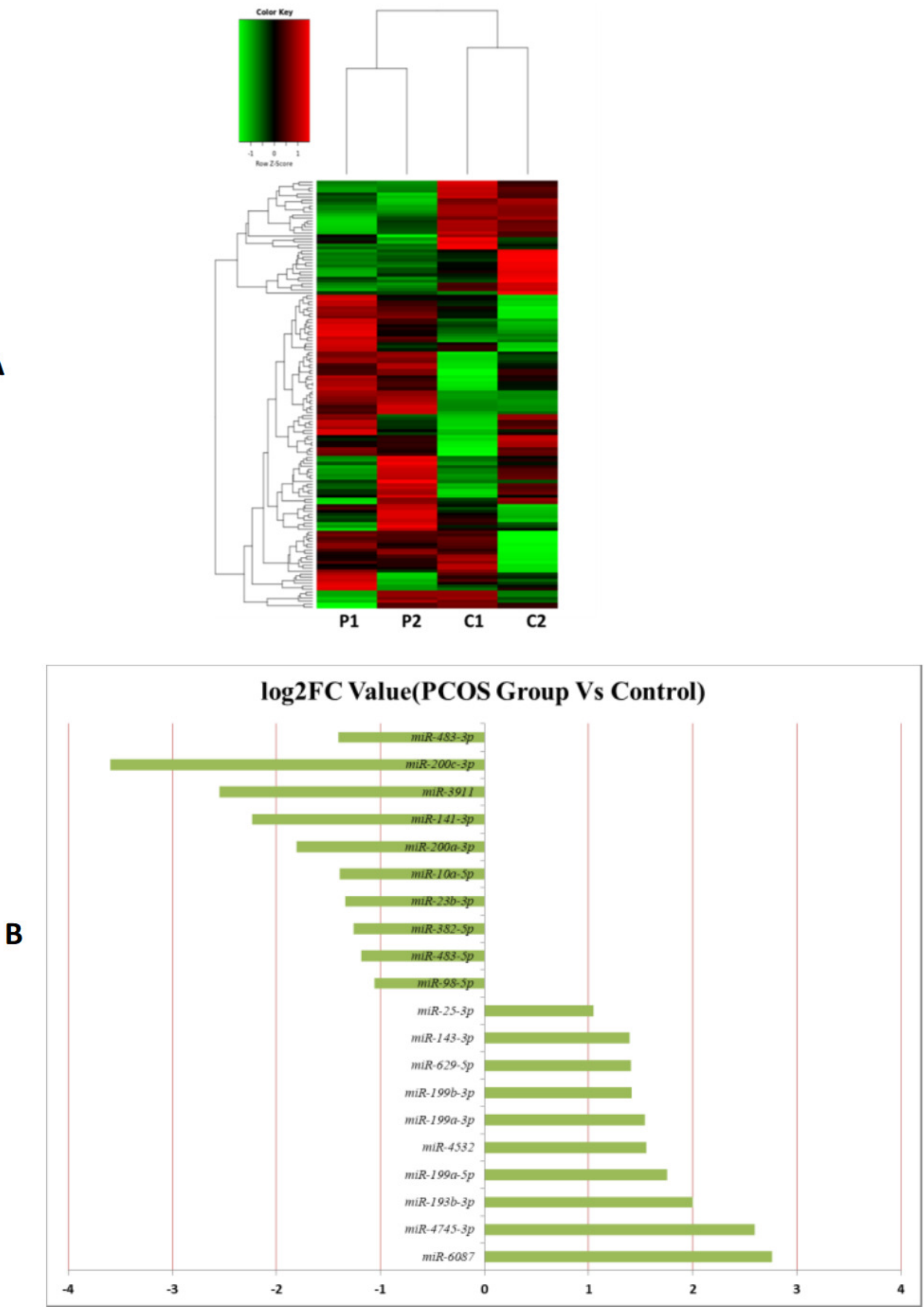
Figure 4

The PiRNA expression analysis of HFF exosomes

(A) Heat map of hierarchical clustering analysis of PiRNA shown in HFF of PCOS group (P1 and P2) and the control group ( $\mathrm{C} 1$ and $\mathrm{C} 2$ ). (B) These are 10 significantly up and down expression PiRNA separately according to their $P$ Value and fold changes $(P<0.05$ and log 2 |(foldchanges) $\mid>1)$. 
A
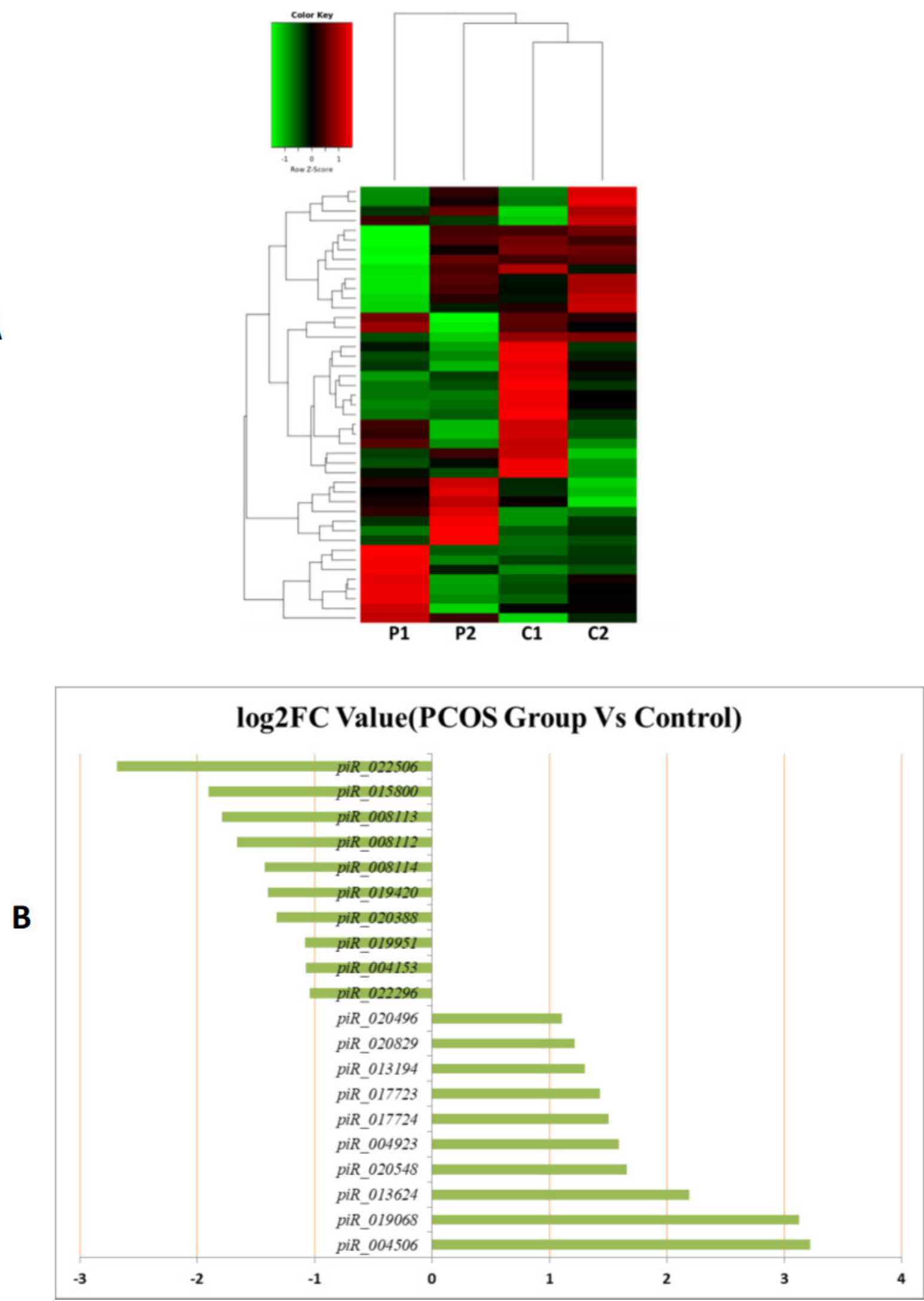


\section{Figure 5}

The tRNA expression analysis of HFF exosomes

(A) Heat map of hierarchical clustering analysis of PiRNA shown in HFF of PCOS group (P1 and P2) and the control group ( $\mathrm{C} 1$ and $\mathrm{C} 2$ ). (B) These are 10 significantly up and down expression tRNA separately according to their $P$ Value and fold changes $(P<0.05$ and log 2 |(foldchanges) $\mid>1)$ 
A
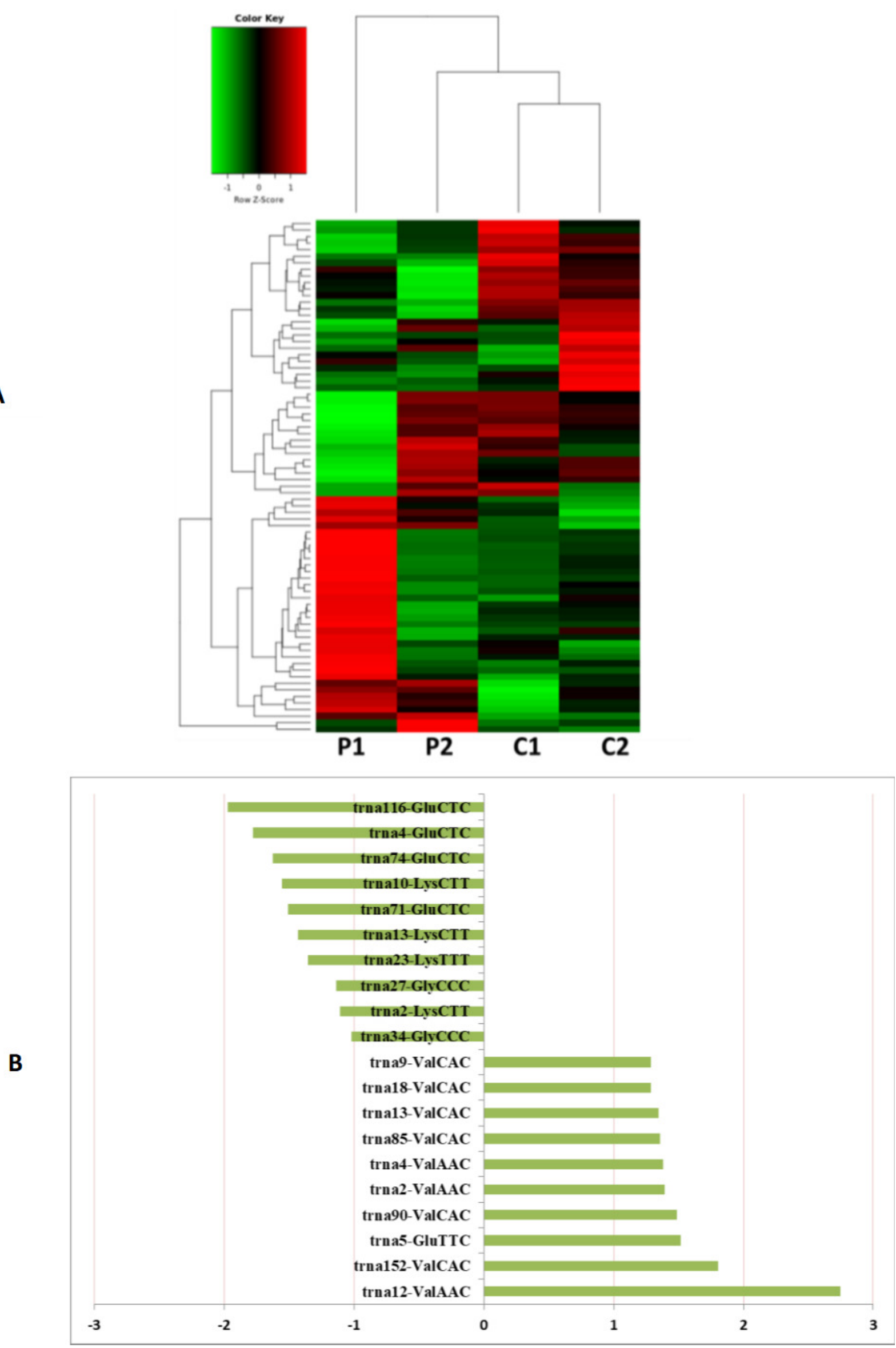
Figure 6

GO function analysis of different expression genes

(A) GO analysis of all the differentially expressed genes included the leftmost Biological Process (BP), the intermediate Molecular Function (MF), and the rightmost Cellular

Component (CC).(B) The scatter plot is a graphical display of $\mathrm{GO}$ enrichment analysis results.

The degree of GO enrichment is measured by Rich factor, $\mathrm{P}$ value and the number of genes enriched in this pathway. Rich factor refers to the ratio of the number of differentially enriched genes to the number of annotated genes in GO. The larger the Rich factor, the greater the degree of enrichment.
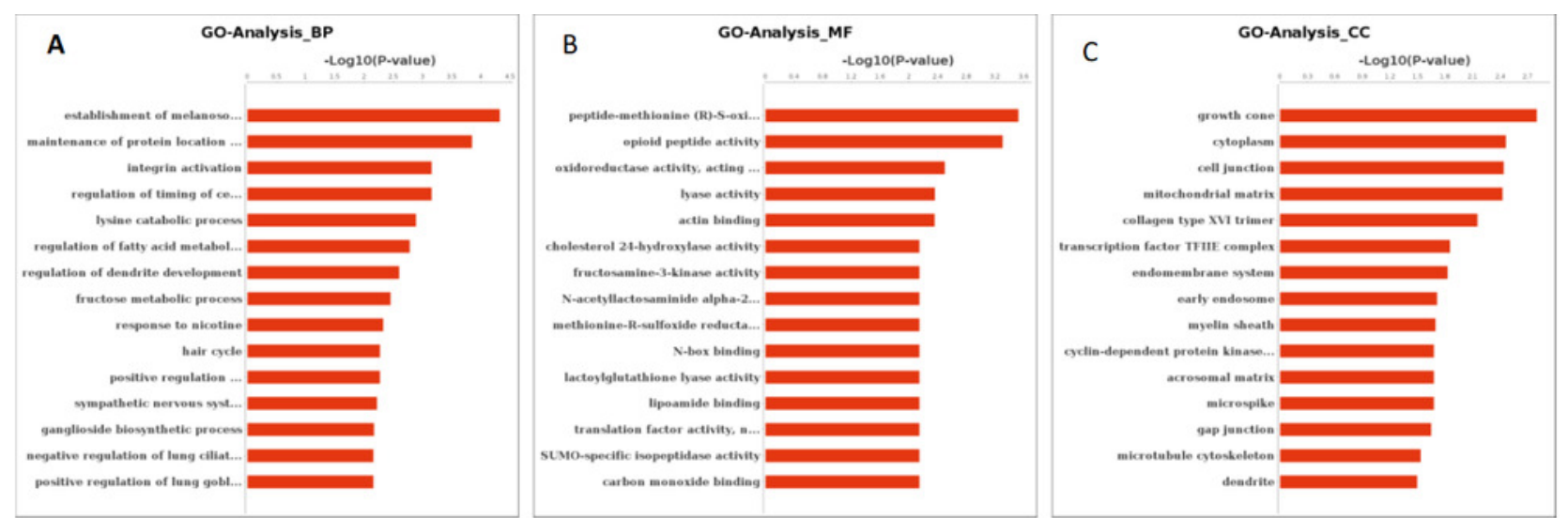

D

Top 20 of GO enrichment

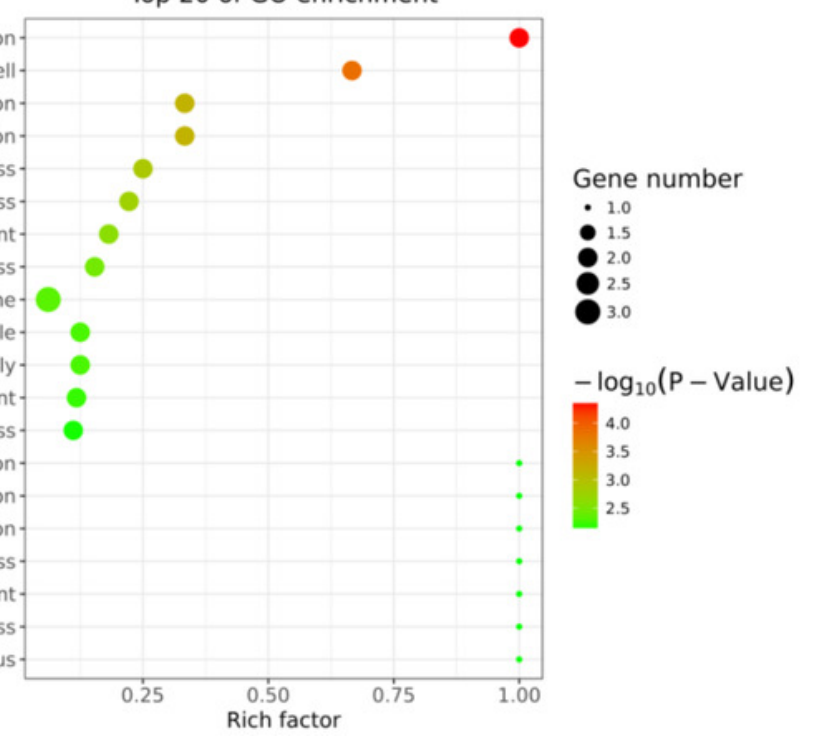




\section{Figure 7}

The pathways of the top 20 entries

(A) Coordinate axis Y: Pathway-Term entry name; coordinate axis X: $\log 10$ (P-Value). Red represents salient items and blue represents non-salient items.(B) The scatter plot is a graphical display of the results of Pathway enrichment analysis. Pathway enrichment is measured by Rich factor, $\mathrm{P}$ value and the number of genes enriched in this pathway. Rich factor refers to the ratio of the number of differentially enriched genes to the number of annotated genes in the Pathway. The larger the Rich factor, the greater the degree of enrichment. 
A

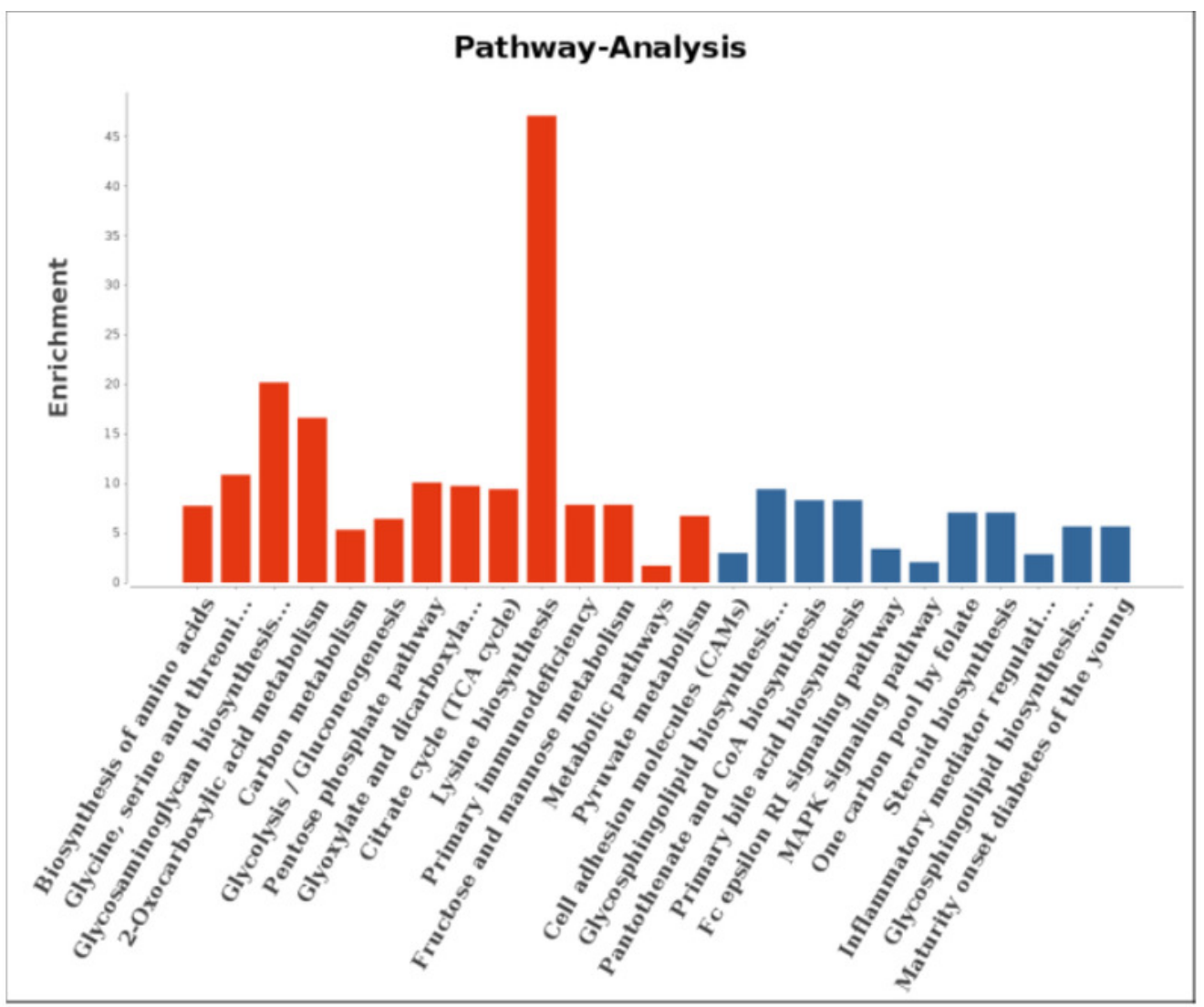

B

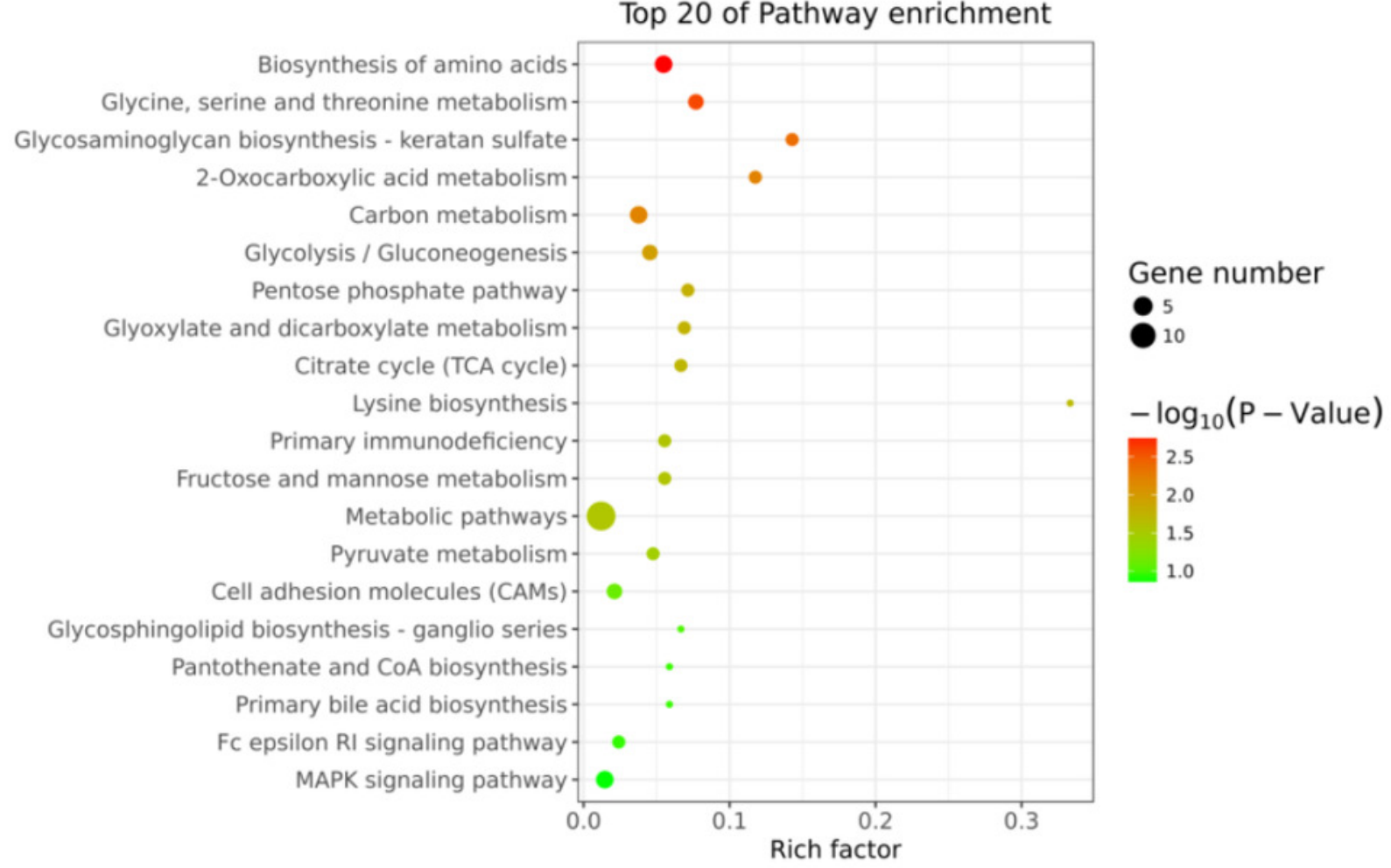

